\title{
Direct solubilization of heterologously expressed membrane proteins by incorporation into nanoscale lipid bilayers
}

\author{
Natanya R. Civjan, Timothy H. Bayburt, Mary A. Schuler, and Stephen G. Sligar
}

BioTechniques 35:556-563 (September 2003)

\begin{abstract}
One of the biggest challenges in the field of proteomics is obtaining functional membrane proteins solubilized and dispersed into a physiologically relevant environment that maintains the spectrum of in vivo activities. Here we describe a system composed of nanoscale self-assembled particles, termed Nanodiscs ${ }^{\mathrm{TM}}$, which contain a single phospholipid bilayer stabilized by an encircling membrane scaffold protein (MSP). Using microsomal membranes of baculovirus-infected Spodoptera frugiperda (Sf9) insect cells overexpressing an N-terminally anchored cytochrome $P 450$ monooxygenase (P450), we demonstrate that target membrane proteins can be directly solubilized and incorporated into distinct populations of Nanodiscs, which can be separated by size chromatography. We show that formation of these Nanodiscs from insect cell membranes allows for the compartmentalization into soluble nanostructures that provide a natural membrane bilayer that avoids the aggregation of membrane proteins often encountered in other reconstitution procedures. Lipid composition analysis and substrate binding analysis of size-fractionated Nanodiscs arrayed in microtiter plates further demonstrates that the Nanodisc system effectively disperses the overexpressed membrane protein into monodispersed bilayers containing biochemically defined lipid components and the target protein in its native form suitable for sensitive high-throughput substrate binding analysis.
\end{abstract}

\section{INTRODUCTION}

Often the critical systems controlling cellular function are located in membrane compartments. Many of these membrane protein assemblies represent important pharmaceutical targets that have been difficult to isolate in soluble and active form because particular phospholipid environments are often essential for maintaining optimal enzymatic turnover or receptor binding activity. Several pharmacologically significant examples indicate specific phospholipid requirements for individual enzymes and receptors, which are perturbed by detergents typically used to solubilize membrane proteins. Examples include the human $\beta$-adrenergic receptor, which requires neutral lipids for efficient receptor hormone response (1), and the human cytochrome P450 monooxygenase (P450) superfamily, which requires several phospholipid types for efficient drug metabolism (2). An inability to faithfully reconstitute the lipid requirements of detergent-solubilized protein in purified systems can, and often does, affect the measured activity of these enzymes. One of the most widely used alternatives for characterization of these native proteins involves the subfractionation of natural cellular membranes and incorporation into micron-sized liposomes. However, liposomes are compromised by thermodynamic instability, size heterogeneity, and sequestration of target membrane proteins on the solvent-inaccessible side of the bilayer $(3,4)$. Other convenient methods for obtaining large quantities of soluble functional membrane proteins assembled in phospholipid bilayers have not been available, and as a consequence, our molecular understanding of the numerous protein complexes functioning within cell membranes has been hindered. Here we report a rapid method for compartmentalizing heterologously expressed membrane proteins into stable soluble nanometer-scale bilayer structures that avoid many of these previously described limitations.

The key to our described self-as- sembly process is the synthesis of an amphipathic multihelical membrane scaffold protein (MSP) whose hydrophobic faces can associate with the nonpolar interior of a supported phospholipid bilayer and whose polar faces provide favorable interactions with the solvent. Design of this synthetic MSP protein has made use of the extensive lipoprotein literature that shows similar polypeptide amphipathicity stabilizing transient forms of discoidal high-density lipoproteins (HDLs) in the physiological process of reverse cholesterol transport $(5,6)$. For instance, a variety of biophysical techniques have indicated that apolipoprotein (apo)A-I, the major protein component of human HDL, when mixed with detergent-solubilized phospholipids, will form 10-nm diameter supported lipid bilayers when the detergent is removed (5-8). Recognizing that the native apoA-I molecule contains amino acid sequences that form recognition domains for receptor-mediated internalization as well as a sequence that 
allows for structural plasticity in the formation of the more common spherical form of the human lipoproteins (5), we synthesized a truncated form of apoA-I, termed MSP, that is idealized for bacterial expression, purification, and self-assembly of discoidal lipid nanobilayers (Nanodiscs $^{\mathrm{TM}}$ ) (9). MSP is a synthetic gene containing 200 amino acid residues arranged in amphipathic helices and an $\mathrm{N}$-terminal $\mathrm{His}_{6}$-Factor $\mathrm{X}$ tag sequence. We reasoned that this Nanodisc system could provide a simple and ideal means for high-throughput solubilization and analysis of heterologously expressed membrane proteins, if these proteins could be directly incorporated into Nanodiscs without the need for prepurification of the target. Here we report the methodologies for transferring membrane-bound proteins directly from partially fractionated cellular membranes into soluble nanostructures.

To demonstrate this assembly process using heterologously expressed membrane proteins, Spodoptera frugiperda (Sf9) insect cells were transfected with a recombinant baculovirus construct containing cDNA sequences for our target membrane protein. The particular protein chosen for this analysis was CYP6B1, a P450 isolated from the black swallowtail (Papilio polyxenes), which is responsible for this insect's ability to bind and detoxify plant psoralen derivatives encountered in its environment $(10,11)$. The spectral properties of this and other P450s are optimal for monitoring the assembly of functional MSP-protein complexes, because they allow the concentration and integrity of the CYP6B1 target protein to be readily followed throughout the solubilization and fractionation procedures (12). The self-assembly process described below indicates that this heterologously expressed P450 protein can be effectively dispersed into soluble nanoscale lipid bilayer cassettes, sizefractionated, and arrayed in a format suitable for high-throughput analysis of substrate binding.

\section{MATERIALS AND METHODS}

\section{Protein Expression and Purification}

Expression and purification of MSP was carried out as previously described (9).

\section{Preparation of Microsomes}

Construction of the recombinant CYP6B1 baculovirus expression vector and infection of Sf9 was performed as previously described (10). Typically, 32 plates containing $6 \times 10^{7}$ baculovirus-infected cells each [multiplicity of infection (MOI) of 2] were collected 72 $\mathrm{h}$ postinfection. Microsomal membranes were homogenized in $2 \mathrm{~mL}$ grinding buffer ( $\mathrm{pH}$ 7.8) composed of $0.1 \mathrm{M}$ sodium phosphate buffer, pH 7.8, 1.1 mM EDTA, $20 \%$ glycerol, $0.5 \mathrm{mM}$ phenylmethylsulfonyl fluoride (PMSF), $0.1 \mathrm{mM}$ dithiothreitol (DTT), and $5 \mu \mathrm{g} / \mathrm{mL}$ (w/v) leupeptin. Membranes were frozen in liquid nitrogen and stored at $-80^{\circ} \mathrm{C}$.

\section{Nanodisc Assembly}

The lipid concentration of the membranes was determined using a $\mathrm{BCA}^{\mathrm{TM}}$ protein assay kit (Pierce Chemical, Rockford, IL, USA) and a 1:1 mass relationship of protein/lipid in the membranes. The membranes were detergent-solubilized with $0.5 \mathrm{M}$ cholic acid and mixed with MSP in the approximate ratio of 1 : 100:200 for MSP/lipid/detergent. Typically, reconstitution samples included approximately $100 \mathrm{nmol}$ scaffold protein, $10 \mu \mathrm{mol}$ lipid, and $20 \mu \mathrm{mol}$ cholate and were pre-incubated for $1.5 \mathrm{~h}$ at $4^{\circ} \mathrm{C}$. Detergent was removed by incubating with Bio-Beads ${ }^{\circledR}$ SM-2 Adsorbent from (BioRad Laboratories, Hercules, CA, USA) (approximately $0.4 \mathrm{~g}$ Bio-Beads $/ 1 \mathrm{~mL}$ reconstitution mixture) for $1.5 \mathrm{~h}$ at $4^{\circ} \mathrm{C}$ followed by centrifugation at $11,750 \times g$ for 5 min. His 6 -tagged MSP particles were purified by incubating with $1 \mathrm{~mL}$ of Ni-NTA agarose (Qiagen, Valencia, CA, USA) per $7.5 \mathrm{~g}$ of $\mathrm{His}_{6}$-tagged MSP for $1 \mathrm{~h}$ at $4^{\circ} \mathrm{C}$, followed by centrifugation at $11,750 \times g$ for $5 \mathrm{~min}$. MSP particles bound to the Ni-NTA agarose were washed with three sequential resin volumes of $0.1 \mathrm{M}$ sodium phosphate buffer (pH 7.4) containing $0.3 \mathrm{M} \mathrm{NaCl}$, $0.15 \mathrm{M} \mathrm{NaCl}$, and no $\mathrm{NaCl}$, respectively. To maintain the integrity of the CYP6B1 protein, MSP particles were eluted with $0.1 \mathrm{M}$ sodium phosphate buffer ( $\mathrm{pH} 7.4)$ containing $0.25 \mathrm{M}$ EDTA rather than the $50 \mathrm{mM}$ imidazole used in previous MSP purifications.

\section{Gel Electrophoresis}

For sodium dodecyl sulfate polyacrylamide gel electrophoresis (SDS-PAGE), microliter samples were separated on PhastGel $^{\mathrm{TM}}$ Gradient 8-25 (Amersham Biosciences, Piscataway, NJ, USA) and stained with Coomassie ${ }^{\circledR}$ Brilliant Blue.

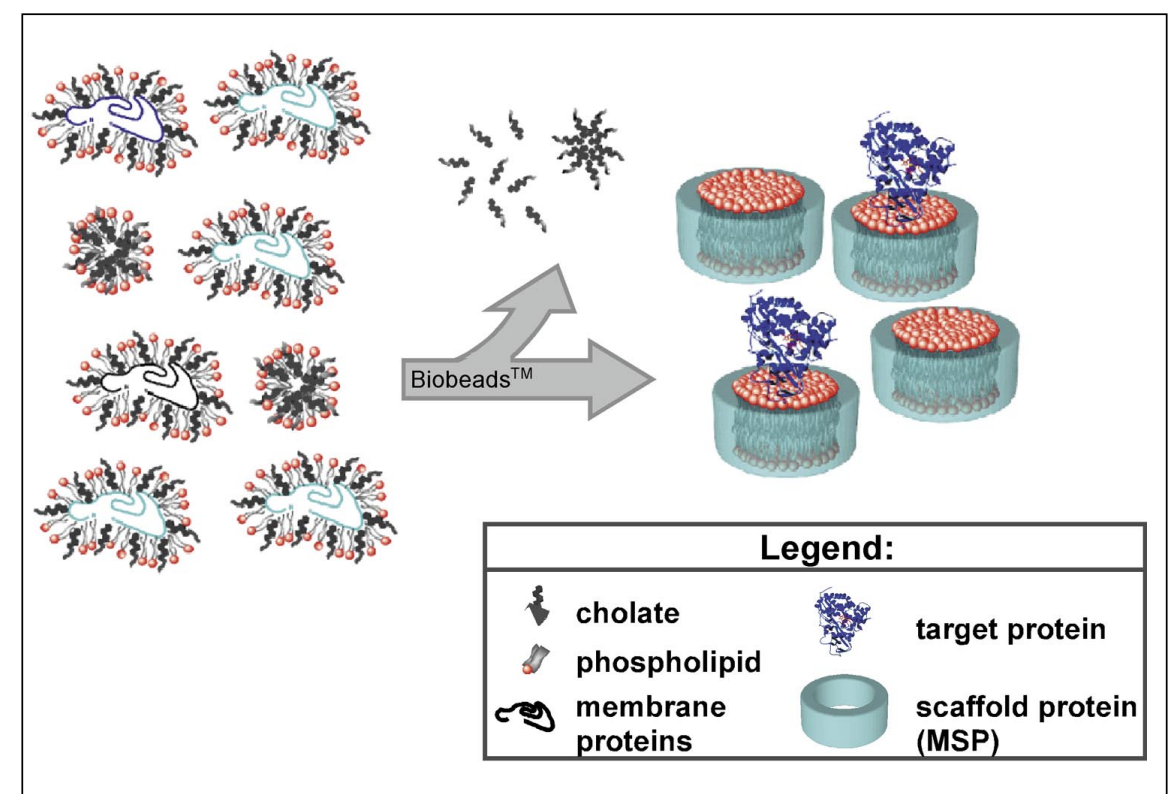

Figure 1. Formation of nanoscale-supported lipid bilayers (Nanodiscs) through self-assembly. A cell membrane preparation containing the target membrane protein is solubilized with detergent in the presence of membrane scaffold protein (MSP). Upon removal of the detergent, by dialysis or Bio-Beads, a soluble MSP-supported Nanodisc is formed with the target incorporated into the resulting phospholipid bilayer. 


\section{Sizing Column Purification}

The nickel affinity-purified sample mixture was injected onto a Superdex ${ }^{\mathrm{TM}}$ 200 HR10/30 gel filtration column (Amersham Biosciences) equilibrated in $0.1 \mathrm{M}$ sodium phosphate buffer ( $\mathrm{pH} 7.4)$ at a flow rate of $0.5 \mathrm{~mL} / \mathrm{min}$. Fractions containing CYP6B1 were concentrated using a Centricon ${ }^{\circledR}$ YM-30 centrifugal filter device (Millipore, Billerica, MA, USA) and reinjected onto the Superdex 200 HR10/30 gel filtration column under the same buffer conditions.

\section{Lipid Extraction}

Lipids were extracted by the Folch method (13), where the sample was homogenized with 2:1 chloroform/methanol $(\mathrm{v} / \mathrm{v})$ and washed with one-fourth volume $0.88 \% \mathrm{KCl}$ in water. The solution was mixed vigorously, and the phases were completely separated by centrifugation $(3000 \times g)$ for $5 \mathrm{~min}$. The organic layer was dried using nitrogen. The lipids were resuspended in a small amount of chloroform and stored at $-20^{\circ} \mathrm{C}$.

\section{Thin-Layer Chromatography}

Samples were spotted onto preparative silica gel stationary phase thin-layer chromatography (TLC) plates purchased from EMD Chemicals (Gibbstown, NJ, USA) alongside phospholipid stan-

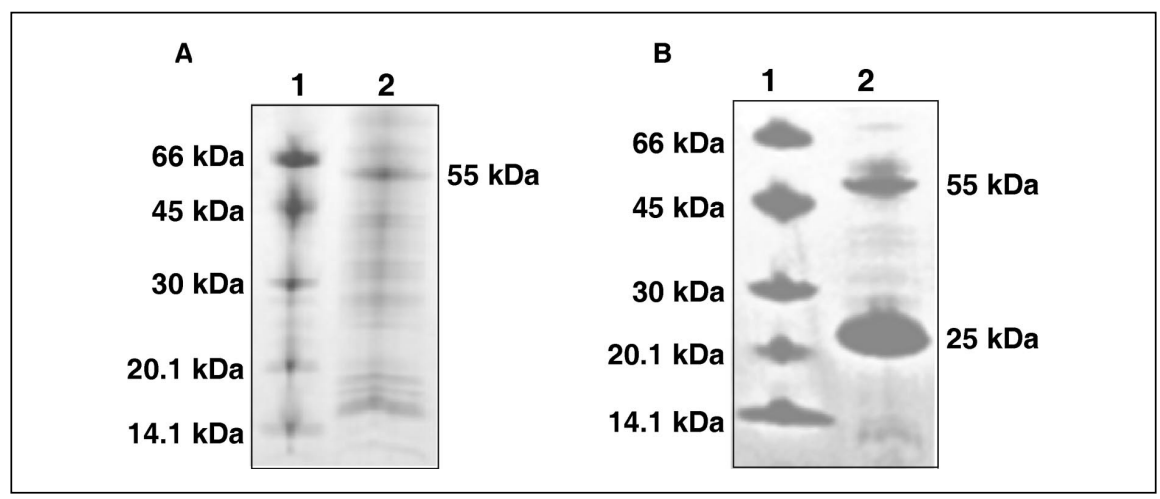

Figure 2. One-dimensional sodium dodecyl sulfate polyacrylamide gel electrophoresis (SDSPAGE) of Nanodisc mixture. (A and B) Lanes 1, low molecular weight markers. (A) Lane 2, Spodoptera frugiperda (Sf9) insect cell membranes infected for the overexpression of CYP6B1. The band at $55 \mathrm{kDa}$ represents the overexpressed target membrane-bound protein. (B) Lane 2 illustrates the nickel affinity-purified Nanodisc mixture assembled from Sf9 insect cell membranes overexpressing CYP6B1. MSP and CYP6B1 run at molecular weights of 25 and $55 \mathrm{kDa}$, respectively.
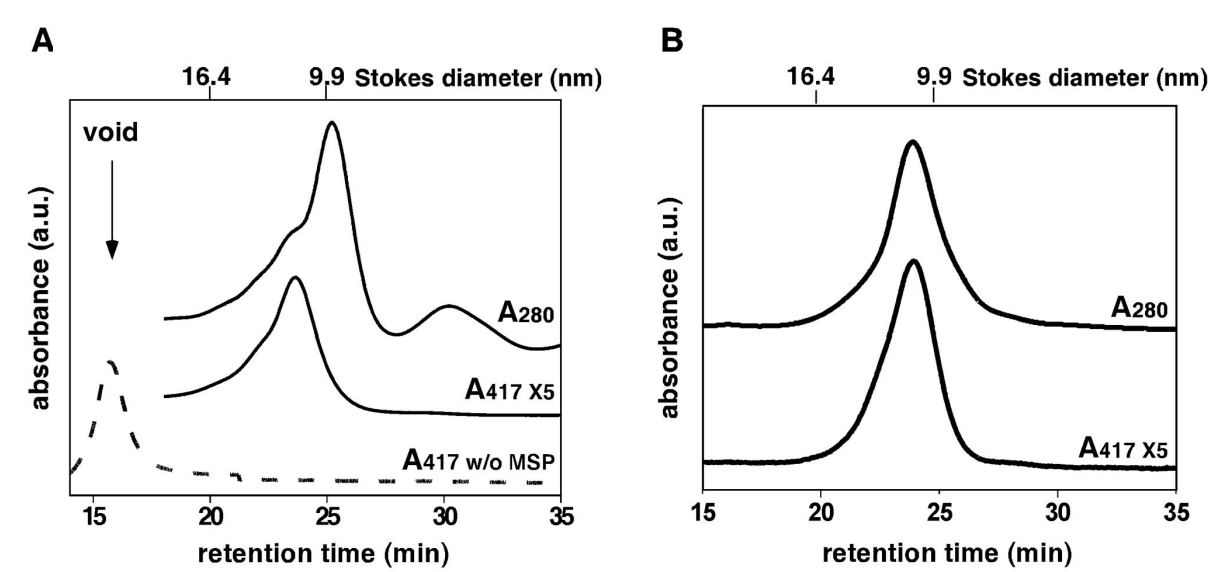

Figure 3. Size-exclusion chromatography of Nanodiscs containing a heterologously expressed cytochrome P450, CYP6B1. The target protein is incorporated into the Nanodisc through the simple self-assembly process described in the text. (A) Chromatogram showing the size separation of the reconstituted particles (Superdex 200). Dotted line shows size separation of a membrane sample in the absence of membrane scaffold protein (MSP) showing the presence of high molecular weight nonspecific aggregates. (B) Rechromatogram of the CYP6B1-containing fraction demonstrating the homogeneity of the self-assembled CYP6B1 bilayer structure. dards purchased from Avanti (Alabaster, AL, USA) and developed using a mobile phase of chloroform/methanol/ ammonium hydroxide (65:25:4). TLC plates were exposed to iodine vapor for visualization, scanned using a ScanJet (Hewlett Packard, Palo Alto, CA, USA), and quantified on a Macintosh ${ }^{\circledR}$ computer using the public domain National Institutes of Health (NIH) Image program developed at the NIH (available at http://rsb.info.nih.gov/nih-image/).

\section{Substrate Binding}

The CYP6B1-containing population of Nanodiscs collected after Superdex size fractionation was concentrated to an enzyme concentration of $50 \mathrm{nM}$. A microtiter plate was arranged with wells A1-A5 and B1-B5 each containing 200 $\mu \mathrm{L}$ Nanodisc samples and wells $\mathrm{C} 1-\mathrm{C} 5$ each containing $200 \mu \mathrm{L}$ buffer $(0.1 \mathrm{M}$ sodium phosphate, $\mathrm{pH}$ 7.4). To rows $\mathrm{A}$ and $\mathrm{C}$, a $20 \mathrm{mM}$ stock concentration of xanthotoxin (Sigma, St. Louis, MO, USA) in methanol was added to yield final concentrations of 0 (column 1), 10 (column 2), 20 (column 3), 50 (column 4), and $150 \mu \mathrm{M}$ (column 5). This dilution was such that the total organic solvent content did not exceed $1 \%$ when added to the Nanodisc samples. To row B, $0,0.1$, $0.2,0.5$, and $1.5 \mu \mathrm{L}$ methanol were added. The contents of each microtiter well were scanned at 1-nm increments using a SpectraMax ${ }^{\circledR}$ Plus microplate spectrophotometer (Molecular Devices, Sunnyvale, CA, USA) and were corrected for the background buffer absorbance (defined in row $\mathrm{C}$ ) and Nanodisc absorbance (well A1).

\section{RESULTS AND DISCUSSION}

\section{Assembly of Nanodises with Heterologously Expressed Membrane Proteins}

To adapt MSP technology to a format compatible with proteomic analysis of heterologously expressed membrane proteins, membranes from $\mathrm{Sf} 9$ cells, overexpressing CYP6B1, were completely solubilized with detergent in the presence of the scaffold protein, MSP. Removal of the detergent 


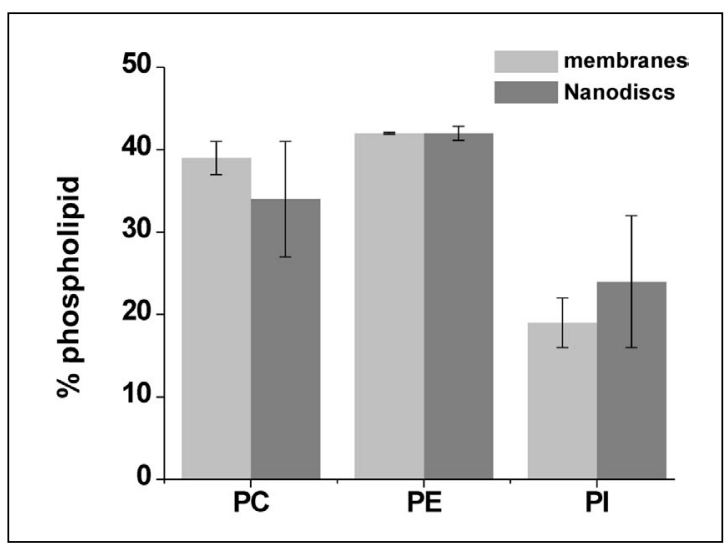

Figure 4. Phospholipid content of starting membrane preparation in the resulting soluble Nanodisc bilayers. Vertical bars represent phospholipid type determined from three replicate samples of starting membranes or self-assembled Nanodiscs. PC, phosphatidylcholine; PE, phosphatidylethanolamine; PI, phosphatidylinositol.

using Bio-Beads initiated self-assembly, allowing for the incorporation of the membrane protein population into MSPsupported phospholipid nanobilayers, as outlined in Figure 1. The MSP-containing particles were subsequently isolated using a nickel-chelating resin to bind the $\mathrm{His}_{6}$-tag on the $\mathrm{N}$ terminus of the scaffold protein. Analysis of the affinity-purified soluble nanobilayers by denaturing PAGE confirmed the presence of the CYP6B1 target protein as well as an array of endogenous proteins present in the original $\mathrm{Sf} 9$ cell membranes (Figure 2). The nickel affinity-purified sample was fractionated by size-exclusion chromatography (Figure 3A) and analyzed by absorbance at $417 \mathrm{~nm}$ to identify a $10-\mathrm{nm}$ fraction containing over $90 \%$ of the solubilized heme-containing target protein. Size-exclusion chromatography of CYP6B1-expressing Sf9 cell membranes, which were treated and fraction-

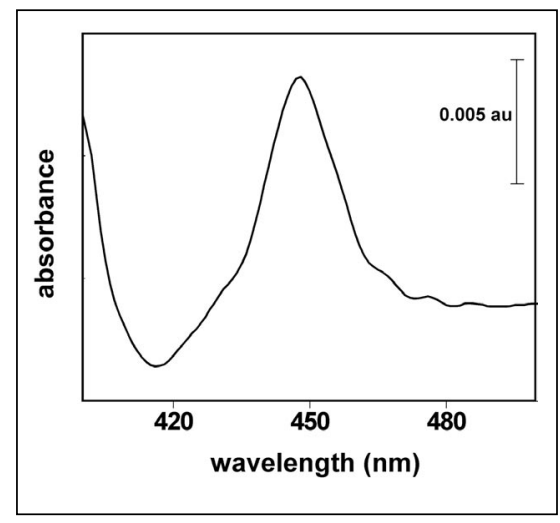

Figure 5. Optical spectrum of carbon monoxide-bound cytochrome $P 450$ with a peak at $450 \mathrm{~nm}$. Such spectra indicate a correct thiolate heme ligation and no evidence for the presence of an inactive P420 form of the cytochrome in the solubilized membrane bilayer system.

ated in the absence of the MSP, shows that the target elutes as large nonspecific aggregates (Figure 3A, dotted line). The homogeneity of the MSP-supported Nanodiscs generated is dependent on the 
identity of lipid and its ratio of lipid to the amount of MSP used in the reconstitution procedure (9). Our analysis of MSP disks assembled with the natural lipid pool from Sf9 insect cell membranes indicates other size populations in the initial nickel affinity-purified Nanodiscs (Figure 3A). These variations are due to the difficulty in determining a priori the precise concentration of MSP protein ideally matched to the lipid composition in membrane preparations expressing variable amounts of the $\mathrm{P} 450$ protein and to the significant size distribution of the endogenous membrane proteins that are also assembled into nanostructures in this process. These other size classes represent nonspecific aggregates that are easily separated from the approximately 10-nm diameter nanobilayer assemblies. Size-fractionated populations of Nanodiscs containing the P450 target protein are uniform and stable through refractionation on the Superdex 200 sizing column. The final CYP6B1-containing population displays a stoichiometry of approximately one CYP6B1 protein per 10 Nanodiscs (Figure 3B).

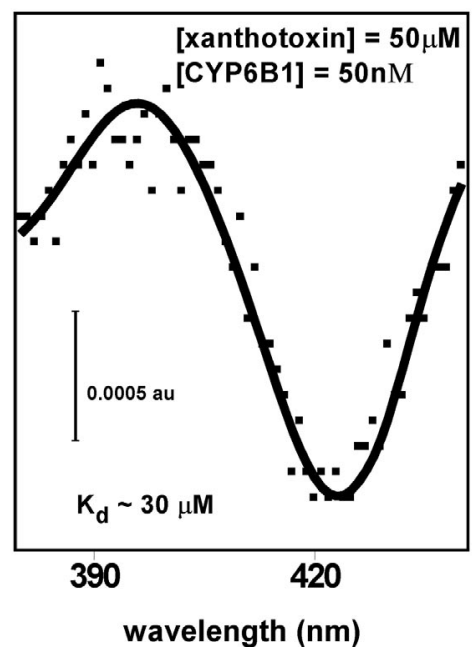

Figure 6. Ligand binding to CYP6B1 incorporated into Nanodisc membrane bilayers. The characteristic type-I binding spectra (decrease in substrate low-spin cytochrome with absorbance at approximately $417 \mathrm{~nm}$ and concomitant increase in the high-spin fraction absorbing at approximately $390 \mathrm{~nm}$ ) is obtained in microtiter plates using a high-throughput plate reader following incremental addition of the environmental furanocoumarin xanthotoxin. A dissociation constant of roughly $30 \mu \mathrm{M}$ is obtained (data not shown).

\section{Lipid Composition of Nanodiscs Formed with Natural Cell Membranes}

The application of MSP technology to the assembly of nanobilayers from natural biological membranes provides a unique opportunity for the direct isolation of membrane proteins from cells and their solubilization and dispersal into a system that provides a close mimic to the native cell environment. To further clarify the extent to which the phospholipid content of the isolated Nanodiscs mimics that of the original Sf9 microsomal membranes, the nickel affinity-purified and size-fractionated nanostructures assembled with $\mathrm{Sf} 9 \mathrm{mi}-$ crosomal membranes were analyzed by TLC. Comparisons of these Nanodisc phospholipid populations with the major phospholipid types found in insect cell membranes, which are phosphatidylcholine, phosphatidylinositol, and phosphatidylethanolamine (14) (Figure 4 ), clearly indicates that the phospholipid composition of endogenous Sf9 microsomal membranes is preserved in assembled Nanodiscs.

\section{Integrity of Target Membrane Protein after Nanodisc Solubilization}

To determine the integrity of the membrane protein assembled into Nanodiscs, CYP6B1-containing nanostructures were assayed by reduction of the iron and binding of carbon monoxide (CO), which monitors, via an absorbance maximum at $450 \mathrm{~nm}$, the quantity of protein that is intact and correctly configured for P450-mediated catalysis (Figure 5) (12). This spectral assay indicates a clear absence of absorbance at $420 \mathrm{~nm}$, which is indicative of a commonly observed inactive form of the protein and documents the fact that normally labile proteins, such as $\mathrm{P} 450 \mathrm{~s}$, are incorporated in their native form into Nanodiscs suitable for subsequent fractionation and biochemical analysis. To further demonstrate that the solubilized membrane protein is accessible for binding substrate and suitable for use in high-throughput optical analysis, the binding of xanthotoxin, one of several furanocoumarin substrates metabolized by this $\mathrm{P} 450$, to CYP6B1-containing Nanodiscs was analyzed in 96-well microtiter plates using a sample volume of only $200 \mu \mathrm{L}$ Nanodiscs (10 pmol enzyme) and varying concentrations of substrate. The type-I binding spectra (15) obtained at varying concentrations of xanthotoxin (Figure 6) show an absorbance shift from 420 to $390 \mathrm{~nm}$ that is characteristic of substrates effectively displacing water as the sixth ligand to the heme iron in the P450 catalytic site and converting the iron from low spin to high spin. These data clearly illustrate that CYP6B1's ability to bind substrate is maintained throughout the Nanodisc assembly and subsequent fractionation process.

\section{CONCLUSION}

In summary, we have described an important tool for the study of membrane protein targets as well as the complicated multicomponent assemblies present in cellular bilayers. When coupled with our ability to express individual cloned P450s or other membrane proteins in the frequently used baculovirus, yeast, and mammalian expression systems, this technology presents the opportunity to display single membrane proteins supported in native membrane bilayers in the development of biochemical methodologies previously restricted to soluble proteins. The ability to bind substrates, inhibitors, and other interacting molecules with these solubilized membrane proteins using sensitive optical difference spectra in microtiter plates has significant potential for development of high-throughput screening methods for many different types of membrane proteins. The fact that the Nanodisc solubilization procedures may be applied nonspecifically to all membrane proteins bodes well for the development of this technology as a means for solubilization and fractionation of many pharmacological target proteins directly out of cellular membranes. Coupled with the histidine tag on the MSP molecule, this technology also presents the means to immobilize target proteins on surfaces suitable for high-throughput screening. The generation of soluble, homogeneous, and monodispersed preparations of 
membrane proteins incorporated into a native bilayer structure enables the techniques for structural and functional characterizations to be applied to this important class of biological macromolecules.

\section{ACKNOWLEDGMENTS}

This research was supported by grants from the National Science Foundation (MCB 0115068) and the National Institutes of Health (R01GM33775, R21GM63574, R01GM50007). The authors would like to thank Ms. Jin-yi Yang for supplying microsomal membranes and Ms. Yelena Grinkova for valuable discussions.

\section{REFERENCES}

1.Kirilovsky, J., S. Steiner-Mordoch, Z. Selinger, and M. Schramm. 1985. Lipid requirements for reconstitution of the delipidated beta-adrenergic receptor and the regulatory protein. FEBS Lett. 183:75-80.

2.Imaoka, S., Y. Imai, T. Shimada, and Y. Funae. 1992. Role of phospholipids in reconstituted cytochrome P450 3A form and mechanism of their activation of catalytic activity. Biochemistry 31:6063-6069.

3.Angrand, M., A. Briolay, F. Ronzon, and B. Roux. 1997. Detergent-mediated reconstitution of a glycosyl-phosphatidylinositolprotein into liposomes. Eur. J. Biochem. 250: 168-176.

4.Savelli, G., N. Spreti, and P. Di Profio. 2000. Enzyme activity and stability control by amphiphilic self-organizing systems in aqueous solutions. Curr. Opin. Colloid Interface Sci. 5:111-117.

5.Brouillette, C.G., G.M. Anantharamaiah, J.A. Engler, and D.W. Borhani. 2001. Structural models of human apolipoprotein A-I: a critical analysis and review. Biochim. Biophys. Acta 1531:4-46.

6.Jonas, A. 1986. Reconstitution of high-density lipoproteins. Methods Enzymol. 128: 553-582.

7.Bayburt, T.H., J.W. Carlson, and S.G. Sligar. 1998. Reconstitution and imaging of a membrane protein in a nanometer-size phospholipid bilayer. J. Struct. Biol. 123:37-44.

8.Carlson, J.W., A. Jonas, and S.G. Sligar. 1997. Imaging and manipulation of high-density lipoproteins. Biophys. J. 73:1184-1189.

9.Bayburt, T.H., Y.V. Grinkova, and S.G. Sligar. 2002. Self-assembly of discoidal phospholipid bilayer nanoparticles with membrane scaffold proteins. Nano Lett. 2:853-856.

10.Chen, J.S., M.R. Berenbaum, and M.A. Schuler. 2002. Amino acids in SRS1 and SRS6 are critical for furanocoumarin metabolism by CYP6B1v1, a cytochrome P450 monooxygenase. Insect Mol. Biol. 11:175-186.
11.Ma, R., M.B. Cohen, M.R. Berenbaum, and M.A. Schuler. 1994. Black swallowtail (Papilio polyxenes) alleles encode cytochrome $\mathrm{P} 450 \mathrm{~s}$ that selectively metabolize linear furanocoumarins. Arch. Biochem. Biophys. 310:332-340.

12.Omura, T. and R. Sato. 1964. The carbon monoxide-binding pigment of liver microsomes. I. Evidence for its hemoprotein nature. J. Biol. Chem. 239:2370-2378.

13.Folch-Pi, J., M. Lees, and G.H.S. Stanley. 1957. A simple method for the isolation and purification of total lipids from animal tissues. J. Biol. Chem. 226:497-509.

14.Marheineke, K., S. Grunewald, W. Christie, and H. Reilander. 1998. Lipid composition of Spodoptera frugiperda (Sf9) and Trichoplusia ni (Tn) insect cells used for baculovirus infection. FEBS Lett. 441:49-52.

15.Estabrook, R.W. and J. Werringloer. 1978. The measurement of difference spectra: application to the cytochromes of microsomes. Methods Enzymol. 52:212-220.

Received 18 March 2003; accepted 13 June 2003.

\section{Address correspondence to: \\ Stephen Sligar \\ Department of Biochemistry \\ University of Illinois \\ 505 S. Goodwin Avenue \\ Urbana, IL 61801, USA \\ e-mail: s-sligar@uiuc.edu}

or

Mary Schuler

Department of Cell and Structural Biology

1201 W. Gregory Drive

University of Illinios

Urbana, IL 61801, USA

e-mail:maryschu@uiuc.edu 